\title{
Linx
}

Revue des linguistes de l'université Paris X Nanterre

64-65|2011

Les genres de discours vus par la grammaire

\section{Genres et grammaire dans l'article premier de la Déclaration universelle des droits de l'homme}

\section{Simon Bouquet}

\section{(2) OpenEdition}

\section{Journals}

Édition électronique

URL : http://journals.openedition.org/linx/1409

DOI : 10.4000/linx.1409

ISSN : 2118-9692

Éditeur

Presses universitaires de Paris Nanterre

\section{Édition imprimée}

Date de publication : 1 juillet 2011

Pagination : 133-154

ISSN : 0246-8743

\section{Référence électronique}

Simon Bouquet, « Genres et grammaire dans l'article premier de la Déclaration universelle des droits de l'homme », Linx [En ligne], 64-65 | 2011, mis en ligne le 01 juillet 2014, consulté le 22 avril 2019. URL : http://journals.openedition.org/linx/1409 ; DOI : 10.4000/linx.1409 


\title{
Genres et grammaire dans l'article premier de la Déclaration universelle des droits de l'homme
}

\author{
Simon Bouquet \\ Université Paris Ouest-C.N.R.S. ITEM-C.N.R.S. MoDyCo
}

\begin{abstract}
A quoi aspirent les innombrables et différentes cultures? Elles aspirent, à partir des données largement différentes de leur expérience historique, anthropologique si l'on veut, elles aspirent à quelque chose, vous l'appelez transcendance, c'est un terme qui me convient tout à fait. C'est l'aspiration vers qui me parait la chose à détecter.
\end{abstract}

Stéphane Hessel (in Bouquet et Hessel, 2012)

Il y faut mieux qu'une connaissance. Il y faut une poétique de cela qui advient sans cesse autour de nous.

Édouard Glissant et Patrice Chamoiseau, L'intraitable beauté du monde.

Notre propos, ici, est de montrer comment l'épistémologie saussurienne - ou, pour mieux dire, l'épistémologie néo-saussurienne - est un cadre permettant l'analyse de la relation entre genres et grammaire. Néo-saussurienne, cette épistémologie n'est pas fondée sur le Cours de linguistique générale mais sur les textes saussuriens originaux ${ }^{1}$. Elle s'oppose d'ailleurs diamétralement au célèbre Cours, en postulant trois thèses liées : (1) la thèse que la description des objets de langue est intégralement concevable sur une base sémiotique ${ }^{2}$; (2) la thèse que cette base sémiotique peut être étendue à la

\footnotetext{
${ }^{1}$ Notamment De l'essence double du langage (in Saussure, 2002).

${ }^{2}$ Cette thèse, regardant la phonologie et la syntaxe, n'est pas postulée par le Cours, contrairement aux textes originaux.
} 
description des objets de parole ; (3) la thèse que l'objet essentiel de la linguistique est, sur cette double base, la description de l'articulation inséparable des objets de langue et des objets de parole ${ }^{3}$. Une telle compréhension de la perspective scientifique saussurienne, ayant pour conséquence de dessiner les lignes d'une refondation herméneutique de la science du langage, y projette aussi l'épistémologie d'une linguistique des genres.

Dans une première partie, nous exposerons cette épistémologie en la résumant dans un ensemble de principes qui la sous-tend ${ }^{4}$. Dans un second temps, nous illustrerons son application à l'analyse sémantique partielle de l'article premier de la Déclaration universelle des droits de l'homme (1948).

\section{Une épistémologie néo-saussurienne pour une linguistique des genres}

\subsection{Principe de scientificité}

Le principe de scientificité, déterminant le caractère proprement épistémologique du programme saussurien, est simple : il postule que la linguistique est une science exacte. En cela, elle doit satisfaire aux critères de scientificité qui sont ceux des sciences exactes: (1) la littéralisation (désignation algébrique de ses objets); (2) la formalisation (écriture formelle de ses lois); (3) la falsifiabilité (caractère réfutable de ces lois). C'est en analysant comment la grammaire comparée - science du son - satisfait à ces trois critères généraux, et en étendant ceux-ci à une linguistique du sens, que Saussure pose ce principe de scientificité, dont le critère de littéralisation est garanti par un principe distinct : le principe de différentialité ( $c f$. infra, 1.6.).

\subsection{Principe d'empiricité}

Science exacte, la linguistique s'applique nécessairement à un objet empirique. En tant que tel, cet objet devra : (1) s'inscrire dans un dispositif d'observation au sein d'une triade observateur/observatoire/objet observable -; (2) être doté de coordonnées spatiotemporelles. L'audace de l'épistémologie saussurienne est de postuler un tel principe, connexe au principe de scientificité, relativement à un objet d'esprit 5 .

Ce principe d'empiricité est opérant car le dispositif d'observation propre à la linguistique est le suivant : (a) l'observateur est un sujet parlant - ou, plus précisément,

\footnotetext{
${ }^{3}$ Cette thèse est énoncée ainsi dans De l'essence double du langage: "Sémiologie $=$ morphologie, grammaire, syntaxe, synonymie, rhétorique, stylistique, lexicologie, etc., le tout étant inséparable » (Saussure, 2002:45). Elle contredit le postulat, fameux mais apocryphe, concluant le Cours de linguistique générale : «La linguistique a pour unique et véritable objet la langue envisagée en elle-même et pour elle-même ».

${ }^{4}$ Pour un exposé plus détaillé des présents principes, of. Bouquet, 2012.

${ }^{5}$ C'est parce qu'elle définit comme objet d'esprit le phonème - unité bien identifiable du signifiant et objet d'une science exacte (la grammaire comparée) - et parce qu'elle conçoit, au plan du sens, les unités du signifié comme relevant de correspondances terme à terme avec des compositions d'unités de signifiant, que cette épistémologie, déliant l'analyse du sens de toute référence à un référent tiers, peut postuler qu'un objet d'esprit est passible d'une science exacte. - Dans la terminologie de son époque, Saussure qualifie l'objet de la linguistique comme « un objet de nature concrète bien que purement spirituel » (CLG/E 1.44.263, cours de linguistique générale de 1910-1911 - Saussure 1968 : 44).
} 
l'esprit d'un sujet parlant - ; (b) l'observatoire est, semblablement, l'esprit de ce sujet parlant - doué de sa compétence de locuteur d'une langue, ainsi que de l'aptitude, pouvant être dite métalinguistique, à observer un fait langagier - ; (c) l'objet observable est une séquence de langage, prenant également place, à fin d'observation, dans l'esprit de ce sujet parlant. Quant aux coordonnées spatio-temporelles assignables à la séquence de langage analysée - c'est-à-dire quant à l'attestation du fait actuel de l'existence spatio-temporelle de cette séquence de langage - elles ne peuvent être rapportées qu'à l'évènement de son interprétation ${ }^{6}$ (par opposition à l'événement de la production de cette séquence - largement opaque au dispositif d'observation décrit, regardant les unités de signifié). C'est d'un tel dispositif et de telles coordonnées spatio-temporelles que relève l'observation d'une séquence de langage analysée, par exemple, dans une publication scientifique de linguistique : cette séquence existe d'abord dans l'esprit de l'auteur de cette publication; ultérieurement, elle existe dans l'esprit de chacun de ses lecteurs. On pourrait juger cette caractérisation du dispositif trop générale car valant pour toutes les sciences; elle s'avère pourtant spécifique sur un point déterminant: dans la généralité des sciences exactes, l'objet observé par l'esprit de l'observateur existe aussi hors de l'espace de l'esprit; ce n'est pas le cas en linguistique.

Le principe d'empiricité ainsi défini implique que l'analyse est reproductible. Et que la linguistique qui l'adopte est par définition une linguistique de l'interprétation.

\subsection{Principe de sémioticité}

Le principe de sémioticité, définissant la nature des objets observables, permet leur identification et leur classification. Selon ce principe, les objets observables sont exclusivement des signes. La notion de «signe » désigne la conjonction de deux éléments distincts : un signifiant et un signifié - cette définition d'un signe biface est donnée par Saussure relativement à la langue; elle se laisse étendre à la parole ( $c f$. infra, 1.5. Principe d'herméneuticité). Les signes sont stabilisés par leur appartenance à un système ; celui-ci est saisi par le point de vue synchronique, considérant la coexistence, dans l'esprit d'un sujet parlant, de tous les signes ressortissant à la compétence linguistique de ce sujet ${ }^{7}$.

Homogènes quant à leur caractère biface et quant à leur caractère compositionnel (cf. infra, 1.4.) les signes de langue appartiennent à trois espèces compositionnelles : celle du phonème (unité minimale de signifiant) et de ses composés; celle du morphème (unité minimale de signifié) et de ses composés; celle de la position syntaxique minimale et de ses composés 8 . Ces trois espèces englobent l'intégralité des

\footnotetext{
${ }^{6}$ Interprétation doit s'entendre ici dans son sens technique en linguistique : comme désignant le fait immédiat de la compréhension (par opposition à la non-compréhension) - fait dont répond par ailleurs le principe de sémioticité.

${ }^{7}$ La langue est « un système de signes totalement indépendant de ce qui l'a préparé et tel qu'il existe dans l'esprit des sujets parlants » (De l'essence double... in Saussure, $2002: 43$ )

${ }^{8}$ Dressant la liste de «toute espèce de signe existant dans le langage », Saussure écrit : « $1^{\circ}$ le signe vocal de tout ordre : signe complet tel qu'un mot, ou un pronom, signe complémentaire comme un suffixe ou une racine, signe dénué de toute signification complète ni complémentaire comme un
} 
objets pouvant être décrits par des grammaires de langue. Ainsi toute grammaire de langue est-elle une grammaire sémiotique ${ }^{9}$. La pertinence d'une description sémiotique de la langue est confirmée par le fait que l'espèce compositionnelle des signes se combine avec leur mode de signification dans la typologie fondamentale icône/indice/symbole de C. S. Peirce. Ainsi, par exemple, des valeurs de sens prises par des phonèmes, dans la poésie par exemple, relèvent-elles de l'iconicité; ainsi, la catégorie grammaticale générale de l'anaphore correspond-elle à une indexicalité intra-segmentale, et la catégorie de la deixis à une indexicalité extra-segmentale.

\subsection{Principe de compositionalité}

Le principe de compositionalité pose que la linéarité d'une séquence de langage, analysée comme la composition de signifiants de langue, conjugue deux types d'articulation :

(1) une articulation interne à chacune des trois espèces compositionnelles (phonème, morphème, position syntaxique) - articulation dans laquelle, semblablement, les unités sémiotiques minimales de chaque espèce compositionnelle se composent en plexus d'unités de la même espèce (ainsi, au palier phonologique, l'unité phonème se compose dans le plexus syllabe qui se compose lui-même dans le plexus polysyllabe; au palier morphologique, l'unité morphème se compose dans des plexus polymorphémiques; au palier syntaxique, le principe chomskyen de «fusion » n'est autre que celui de la composition d'unités sémiotiques syntaxiques dans des plexus sémiotiques syntaxiques);

(2) une articulation des espèces compositionnelles entre elles - articulation dans laquelle les plexus maximaux de chaque espèce compositionnelle constituent l'unité minimale du palier compositionnel supérieur (ainsi : un plexus maximal de phonèmes constitue l'unité minimale du palier du morphème; un plexus maximal de morphèmes constitue l'unité minimale du palier de la position syntaxique).

\subsection{Principe d'herméneuticité}

Pour autant, l'interprétation d'une séquence de langage par un sujet parlant n'est pas le simple produit d'une computation ressortissant au principe de compositionalité. L’interprétation linguistique - et donc sa description par une linguistique de l'interprétation - fait intervenir un autre principe : le principe d'herméneuticité. Ce principe stipule que, dans toute séquence de langage, la valeur des signes de langue (phonèmes, morphèmes, positions syntaxiques) est déterminée par la valeur d'un signe global (ou "signe de parole ») attaché à la totalité de cette séquence - le signifié de ce signe global devant s'entendre non comme la somme des signifiés de langue de la séquence de langage analysée, mais comme un (ou plusieurs) trait(s) de sens spécifique(s) s'étendant à l'intégralité de cette séquence.

"son" déterminé de langue; ou $\left[2^{\circ}\right]$ signe non vocal comme "le fait de placer tel signe devant tel autre" (...)» (De l'essence double... in Saussure, 2002:48). Cette conception d'une sémioticité transversale aux niveaux phonologique, morphologique, lexicologique et syntaxique a échappé aux rédacteurs du Cours de linguistique générale.

9 "Grammatical $=$ significatif $=$ ressortissant à un système de signes $=$ synchronique ipso facto » (CLG/E 1.304.2128, cours de linguistique générale de 1909-10 - Saussure 1968 : 304). 
Le terme de genre - bien établi dans les traditions de la rhétorique et de la théorie littéraire - est précisément employé par Schleiermacher pour désigner l'objet « globalité » dans la détermination du local par le global. Il doit s'entendre ici dans une acception très générale : comme une appellation intuitive pour désigner un trait global de sens, ainsi qu'on l'a défini, pouvant être posé relativement à tout texte écrit ou oral. De fait, la notion de «signe global » correspond aussi assez bien à ce que Wittgenstein désigne comme jeux de langage (en postulant un primat de ces derniers sur la détermination logique du sens). A ces notions philosophiques et de théorie littéraire font écho des objets conceptuels plus ou moins intuitifs, familiers à diverses approches d'analyse textuelle ou discursive contemporaines, mais souffrant d'un déficit épistémologique : discours, type, mode, champ générique, sous-genre, registre, domaine, niveau, isotopie, thème, etc.

L'innovation épistémologique néo-saussurienne, en l'occurrence, consiste à reconcevoir dans une logique sémiotique le thème herméneutique de la détermination du local par le global. Une telle reconception sémiotique donne forme à l'articulation inséparable, requise par Saussure, de la linguistique de la langue et de la linguistique de la parole: en posant la notion de «signe global», il devient possible de traiter les signifiés de langue et les signifiés de parole selon une grammaire homogène ( cf. infra, 1.6. Principe de différentialité) et, partant, de formuler des lois qui décrivent le sens, satisfaisant au principe de scientificité (cf. infra, 1.7. Protocole méthodologique).

\subsection{Principe de différentialité}

Du postulat de la coexistence synchronique des signes dans l'esprit des sujets parlants, Saussure tire pour conséquence une thèse ontologique qui a marqué l'histoire des sciences humaines : les signes du langage n'ont pas d'identité propre; interdépendants au sein d'un système, ce sont des entités purement différentielles. Le principe épistémologique de différentialité est l'expression de cette thèse. Il stipule que la valeur des signes linguistiques peut être désignée sur la seule base de cette différence et de cette interdépendance systémique ${ }^{10}$.

Ce principe de différentialité suffit à poser un critère de littéralisation - premier critère du principe de scientificité - propre à la linguistique : la littéralisation (ou grammaire) de la valeur des signes sera la littéralisation de leur différence. Cette littéralisation prendra, par définition, la forme d'une algèbre du type suivant: soit un corpus de signes $a, b, c, d$; la valeur du signe $a$ dans le corpus est littéralisée ${ }^{11}:\{\sim b, \sim c, \sim d\}$; celle du signe $b:\{\sim a, \sim c, \sim d\}$, etc. - ce principe s'appliquant en général, à un type de signes, ou à toute sous-catégorie ou corpus de signes. En cela que cette littéralisation s'étend aux signes de langue et aux signes de parole de manière homogène - c'est-à-dire : sous une forme algébrique homogène - ce principe de différentialité sous-tend également la possibilité de l'écriture formelle de lois - conformément au deuxième critère du principe de scientificité -, ces lois conjuguant inséparablement, en l'occurrence, le

10 «Les objets [que la linguistique] a devant elle n'ont jamais de réalité en soi, ou à part des autres objets à considérer; n'ont absolument aucun substratum à leur existence hors de leur différence ou en DES différences de toute espèce que l'esprit trouve moyen d'attacher à $L A$ différence fondamentale (...) » (De l'essence double... in Saussure, $2002: 65$ )

${ }^{11}$ Le signe ' $\sim$ signifie ici « n'est pas ». 
point de vue d'une linguistique de la langue et celui d'une linguistique de la parole ( $c f$. infra, Protocole méthodologique).

\subsection{Protocole méthodologique (principe de légalité)}

Aux principes épistémologiques qui ont été énumérés, répond un protocole méthodologique, définissant la forme générale que prendront les lois d'une linguistique de l'interprétation. Ce principe de légalité peut s'énoncer ainsi : pour décrire le sens d'une séquence de langage quelconque, la linguistique de linterprétation opposera cette séquence à une séquence homonyme et décrira, sous la forme de lois littéralisées et formalisées, les corrélations entre nivean sémiotique global et nivean sémiotique local, spécifiques à chacune de ces deux séquences bomonymes et déterminant leur différence d'interprétation ${ }^{12}$.

Le caractère réfutable des lois ainsi définies - troisième critère du principe de scientificité - tient à des tests qui peuvent être réalisés par (sur) des sujets parlants. Ces tests impliquent: (1) un jugement de différence portant sur le sens global des deux séquences considérées (test propre à confirmer que les littéralisations grammaticales du signe global ont un objet réel) ; (2) un jugement d'acceptabilité relatif à des procédures de substitution mettant en évidence les sens différentiels des signes locaux analysés dans chacune des séquences (test confirmant que les grammaires littéralisant ces sens différentiels ont un objet réel).

\footnotetext{
12 Techniquement, l'écriture d'une loi de corrélation dans ce cadre méthodologique suppose un corpus d'interprétation et un corpus de grammaires. Le corpus d'interprétation est composé des deux séquences de langage homonymes (c'est-à-dire: de deux séquences auxquelles un sujet-interprète reconnait, pour un même signifiant, deux sens différents - le rôle joué par l'homonymie tient ici essentiellement au fait que, la loi de corrélation posant, au plan du signifié global et du signifié local, une corrélation de deux variables, cette loi requiert l'assise d'une constante: c'est, au plan du signifiant, le segment phonologique, fondement de l'homonymie, qui remplit cette fonction). Le corpus de grammaires (autrement dit: de littéralisations différentielles ad hoc) comprend: (a) une grammaire différentielle de la valeur dans ce corpus d'interprétation des signifiés locaux variant pour chacune des deux séquences ; (b) une grammaire différentielle de la valeur dans ce corpus d'interprétation des signifiés globaux de ces deux séquences.

Sur ces bases, seront opérées les littéralisations et les formalisations suivantes. Soient deux séquences de langage homonymes [SQ1] et [SQ2]; autrement dit, deux segments phonologiques (notés $[\mathrm{SgPHO}])$ interprétés comme phonologiquement identiques: $[\mathrm{Sg} P H O(\mathrm{SQ} 1)]=[\mathrm{Sg} P H O(\mathrm{SQ} 2)]$, et dont le sens (noté [Sé]), conçu selon un simple jugement de différence, est interprété comme non identique: $[$ Sé(SQ1)] $\neq$ [Sé(SQ2)]. Soit le signifié global (noté [SéGL]) de chacune de ces deux séquences, représenté par un trait différentiel (TrSéGL...): [SéGL(SQ1)] $\neq[$ SéGL(SQ2)]; $[$ TrSéGL(SQ1) : +X] / [TrSéGL(SQ2) : -X]. Soit le signifié d'un signe local (noté [SéLOs]), dont le signifiant appartient à l'homonymie de ces deux textes (phonème(s) - exceptionnellement -, morphème, plexus de morphèmes ou structure syntaxique); ce signifié, affecté d'une variation dans chacune des deux séquences, sera représenté par un trait différentiel $\left(\operatorname{TrSé}^{2} \mathrm{O}_{s} \ldots\right)$ : $\left[\mathrm{SéLO}_{s}(\mathrm{SQ} 1)\right] \neq\left[\mathrm{SéLO}_{s}\left(\mathrm{SQ}_{2}\right)\right] ; \quad\left[\operatorname{TrSéLO}_{s}(\mathrm{SQ1}):+\mathrm{a}\right] /\left[\operatorname{TrSéLO}_{s}\left(\mathrm{SQ}_{2}\right):-\mathrm{a}\right]$. La loi d'interprétation consistera à établir simultanément les deux corrélations suivantes: [TrSéGL(SQ1) : $+\mathrm{X}]=>$ $[\operatorname{TrSéLO} s(\mathrm{SQ} 1):+\mathrm{a}] /[\operatorname{TrSéGL}(\mathrm{SQ} 2):-\mathrm{X}]=>\left[\operatorname{TrSéLO}_{s}(\mathrm{SQ} 2):-\mathrm{a}\right]$.
} 
On peut tenir que ce protocole méthodologique permet la description du sens la plus fine possible, et qu'en cela il confirme l'efficace des principes épistémologiques d'une linguistique néo-saussurienne de l'interprétation et sa valeur de modèle épistémologique pour une linguistique des genres.

\section{Analyse semantique partielle de l'article premier de la Déclaration universelle des droits de l'homme ${ }^{13}$}

La Déclaration universelle des droits de l'homme (ci-après DUDH) est un objet d'analyse particulièrement digne d'intérêt, pour deux raisons liées : $1^{\circ}$ c'est un texte constituant $^{14}$ par excellence, qui crée en outre un genre singulier (en cela même qu'il se présente - par son titre et par sa forme générale - comme un texte juridique, tout en se définissant, dans son préambule, de manière fort peu juridique comme «un idéal commun à atteindre progressivement $\left.»^{15}\right) ; 2^{\circ}$ son universalité fait l'objet de controverses quelque peu enlisées depuis des décennies (la question irrésolue étant : «La Déclaration de 1948 peut-elle, en elle-même, être tenue pour un texte universel ; ou : ne le peut-elle pas, en cela qu'elle est inscrite dans une tradition de pensée marquée du sceau de l'Occident ? $»)^{16}$. Or, c'est précisément sur la base de ces deux particularités que peut se fonder une analyse interprétative différentielle, qui procèdera de l'hypothèse suivante : si l'argumentation théorique de l'universalité semble souvent vouée à une impasse, c'est parce que cette argumentation ne tient généralement compte $1^{\circ} \mathrm{ni}$ du fait sémantique que la DUDH se comprend différemment selon qu'on la lit comme un texte légal ou comme un texte éthique, $2^{\circ}$ ni du fait sémantique que la DUDH peut également se comprendre différemment dans des contextes culturels divers.

\subsection{La DUDH dans les genres "légal " et "éthique " : sémantique des verbes de l'article premier}

L'analyse interprétative, on l'a dit, consiste à établir des corrélations entre des signifiés globaux différentiels (signifiés de genres) et des signifiés locaux (signifiés attachés, en l'occurrence, à des morphèmes) variant dans chacun des genres concernés. On appliquera cette méthode à l'article 1 de la DUDH :

[Première proposition] Tous les êtres humains naissent libres et égaux en dignité et en droits. [Deuxième proposition] Ils sont doués de raison et de conscience [Troisième proposition] et doivent agir les uns envers les autres dans un esprit de fraternité.

\footnotetext{
13 Cette section reprend les éléments d'un article paru dans un ouvrage collectif sous la direction de D. Leeman (Leeman, 2011). Je remercie Danielle Leeman pour ses conseils amicaux lors de l'écriture de cette section.

${ }^{14}$ Sur la notion de « discours constituant », cf. Cossuta et Maingueneau, 1995.

15 «L'Assemblée Générale proclame la présente Déclaration universelle des droits de l'homme comme l'idéal commun à atteindre par tous les peuples et toutes les nations (...) [pour que ceux-ci s'efforcent] d'en assurer par des mesures progressives (...) la reconnaissance et l'application universelles et effectives (...)» (DUDH, Préambule)

${ }^{16}$ Cf. Bouquet, 2011.
} 
Une analyse complète de cet article montrerait que verbes, noms et adjectifs font l'objet d'actualisations sémantiques distinctes dans les genres « loi » et « éthique ». La place manquant pour développer une telle analyse, on se contentera de l'illustrer tout d'abord sur le verbe naissent, puis sur les autres verbes conjugués de cet article 1, dont on examinera successivement la valeur du morphème de conjugaison (présent de l'indicatif) dite encore valeur flexionnelle, et la valeur du morphème radical, dite ici valeur lexicale.

\subsubsection{Signifiés globaux}

On posera donc formellement, correspondant à la séquence des mots de l'article 1 , l'existence de deux textes homonymes :

$1^{\circ}$ un texte légal, dont le signifié global sera noté par le trait [+LOI] (notation différentielle équivalant au trait [-ETHIQUE]) ;

$2^{\circ}$ un texte énonçant un idéal éthique, dont le signifié global sera noté par le trait [+ETHIQUE] (notation équivalant au trait [-LOI]).

Cette grammaire différentielle de genres, construite ad hoc, selon les principes épistémologiques d'une linguistique de l'interprétation, - c'est-à-dire construite relativement au seul corpus constitué par les deux textes homonymes considérés -, sténographie une réalité sociale facilement identifiable.

Le texte légal a pour source une autorité politique, édictant les lois régissant la vie collective, dont la non-observance est normalement passible de sanctions. Il s'agit d'un texte "injonctif » (un ordre), " déontique » (on doit se plier à cet ordre), à valeur future puisqu'il vaut à partir du moment où il est énoncé (c'est ce que l'on peut appeler sa «valeur performative »). Ainsi, par exemple, l'Article 3 de la loi du 10 août 2007 relative à l'autonomie des universités - «Les établissements déterminent par délibérations statutaires du conseil d'administration (...)» - s'applique-t-il à partir du moment où ce texte est adopté.

Le texte éthique, quant à lui, définit, de manière spéculative, des comportements humains comme positifs ou négatifs - des comportements considérés comme tels par une morale, par une autorité religieuse (parlant éventuellement au nom d'une divinité), voire par une coutume - sans pour autant qu'une sanction légale ne soit prévue en cas de comportement non conforme (ainsi, par exemple, le code déontologique du fonctionnaire). Quand bien même il ne formule nullement des lois au sens juridique du terme, le genre éthique présente volontiers ses règles de conduite comme éternelles et universelles : «Il se faut entraider, c'est la loi de nature 》 (La Fontaine).

Pour autant, insistons sur ce point, l'analyse ne se fondera ici

- ni sur une théorie objective des genres de textes (l'opposition trait [+LOI] / [+ETHIQUE] ne valant, comme on l'a signalé, que pour le corpus considéré)

- ni sur une conception de ce que doit être l'interprétation de la DUDH ou sur quelque théorie des fondements du droit, mais sur une observation de ce qu'elle peut être (autrement dit, sur une observation des variations de sens possibles de deux textes homonymes, ces variations étant, dans la perspective d'une linguistique de l'interprétation, la seule réalité pouvant faire l'objet d'une écriture de lois et d'une vérification expérimentale). 


\subsubsection{Corrélations entre les signifiés globaux et la valeur flexionnelle du verbe "naissent"}

Afin de rendre compte de la corrélation entre les valeurs des signifiés globaux, qu'on vient de poser formellement, et la valeur flexionnelle (c'est-à-dire la valeur du temps "présent ») du verbe naissent, la double loi suivante peut être formulée, dans laquelle les notations différentielles de valeurs locales doivent également être considérées comme ad hoc, c'est-à-dire relatives au corpus des deux textes homonymes considérés :

$[+\mathrm{LOI}]=>[+$ performatif légal $]$

[+ÉTHIQUE $]=>[+$ vérité générale $]$

En effet, le genre noté [+LOI] actualise une valeur du temps "présent» du verbe naissent qui peut être notée [+performatif légal] (ou [-vérité générale]) : conformément au genre juridique, ce présent peut être tenu pour un performatif (énoncer une loi est ce qui lui donne « force de loi »), contenant les valeurs injonctive et déontique qui ont été évoquées. Un test de commutation permet d'attester de cette valeur: de même que, dans l'exemple proposé plus haut, la formule « Les établissements déterminent, par délibérations statutaires du conseil d'administration (...) » peut être remplacée salva congruitate par «Les établissements détermineront, par délibérations statutaires du conseil d'administration (...) », de même, dans l'interprétation «légale » de la DUDH, il est possible de remplacer le présent par un futur : Tous les êtres bumains naitront libres et égaux en dignité et en droits.

Dans le genre noté [+ETHIQUE], le présent de naissent prend une valeur différente de la précédente. Cette valeur, bien reconnue par la tradition grammaticale comme celle du "présent de vérité générale », sera donc notée [+vérité générale] (ou [performatif légal]) ; elle confère ici à la DUDH le sens d'un constat : « il est de fait que les hommes naissent libres et égaux $»^{17}$. Un test vérifie cette valeur : de même qu'un présent de vérité générale comme celui de la phrase Les baleines sont des mammiferes ou comme celui de la fable citée ne peut être remplacé salva congruitate par un futur (on n'aura pas: Les baleines *seront des mammiferes ou Il se *faudra entraider, c'est la loi de nature), de même Tous les êtres humains *naitront libres et égaux en dignité et en droits est incompatible avec le genre [+ÉTHIQUE].

\subsubsection{Corrélations entre les signifiés globaux et la valeur lexicale du verbe "naissent "}

Regardant maintenant la valeur lexicale du verbe naissent, la double corrélation suivante entre signifié global et signifié local peut être posée :

$[+\mathrm{LOI}]=>[+$ qualité originaire $]$

$[+$ ÉTHIQUE $]=>[+$ destinée $]$

\footnotetext{
${ }^{17}$ L'éthique est en effet interprétable comme l'énonciation d'une vérité générale - qu'elle constate ou qu'elle recherche. Elle s'oppose en cela au texte de loi ou, en tout cas, au caractère performatif de celui-ci, à l'aune duquel l'énonciation légale, si elle a le pouvoir de faire advenir une contrainte, n'a pas le pouvoir de faire advenir une vérité.
} 
Dans le genre [+LOI], en effet, la valeur lexicale du verbe naissent - considérée dans sa construction syntaxique naître $X$, comme déterminant l'adjectif ou le substantif attribut - peut être notée [+qualité originaire] (ou [-destinée]). Cette valeur est celle que prend le verbe naitre dans des énoncés comme Il est né aveugle. Elle implique que l'adjectif ou le substantif attribut dénote « une qualité possédée, à sa naissance, par un être vivant ». Référant à un fait objectif et daté, et non à une destinée, l'existence de cette valeur différentielle dans la langue est attestée par un test négatif, vérifiant que le terme $X$ ne peut pas être remplacé par pour être $X$ : de même qu'on n'aura pas, salva congruitate, *Il est né pour être aveugle, on n'aura pas, dans le genre [+LOI], *Tous les êtres bumains naissent pour être libres et égaux en dignité et en droits.

Dans le genre [+ETHIQUE], la valeur lexicale du verbe naissent - envisagée semblablement pour la construction naitre $X$ - sera notée [+destinée] (ou [-qualité originaire]). Cette valeur est celle que prend ce verbe dans des tours comme Il est né poète $^{18}$. Quant à la qualité attribuée par l'adjectif ou par le substantif, elle ne se réfère pas à un fait objectif et daté, mais au contraire à un destin et/ou à la clairvoyance de dispositions particulières. Un test positif répond de cette valeur : le terme $X$ dépendant du verbe naitre peut être remplacé par pour être $X$; c'est ainsi que, salva congruitate, on pourra dire Il est né pour être poète et, dans le genre [+ÉTHIQUE] de la DUDH, Tous les êtres bumains naissent pour être libres et égaux en dignité et en droits ${ }^{19}$.

\subsubsection{Isotopies différentielles et valeurs des verbes de l'article 1}

Des traits sémantiques transversaux qu'on peut désigner par le terme d'isotopie ${ }^{20}$, propres à chacun des deux genres considérés, lient entre elles la valeur flexionnelle et la valeur lexicale du verbe naissent :

- dans le genre «légal» une isotopie pouvant être notée [+OBJECTIVITÉ] (ou [-SPÉCULATIVITÉ]) répond du fait qu'une loi, en ce qu'elle est arbitraire et suiréférente, énonce une proposition objective, incontestable ; cette isotopie sous-tend la composition de la valeur flexionnelle [+performatif légal] du verbe naissent (l'acte performatif de promulguer une loi est lui-même un acte objectif, incontestable) avec sa valeur lexicale [+qualité originaire] (une qualité originaire est fait objectif, incontestable);

18 Cette valeur se retrouve dans la construction prépositionnelle de naître avec pour: L'homme est né pour le bonbeur.

${ }^{19}$ Si la distinction des valeurs [+qualité originaire] et [+destinée] se fonde ici sur un sens "propre » de la première et un sens «figuré » de la seconde, l'important, pour l'analyse, est que le test de commutation fait apparaître ces valeurs comme synchroniquement opposées. - Par ailleurs, l'emploi du verbe naître, dans la construction naître $X$, semble plus courant à la fin du XVIII ${ }^{\circ}$ siècle que de nos jours. Or, la Déclaration de 1948, dans son article 1, réécrit le premier article de la Déclaration des droits de l'homme et du citoyen de 1789 : Les hommes naissent et demeurent libres et égaux en droits. Il est possible que la DUDH, par sa notoriété, ait contribué à maintenir un tour déclinant et qu'elle lui ait en outre conféré, dans son contexte, un sens quelque peu hiératique - à la fois figé et incertain susceptible de faire obstacle à la conscience des différences virtuelles, qu'on vient d'analyser, de ses signifiés locaux.

${ }^{20}$ Ce terme est utilisé par A.-J. Greimas et F. Rastier, qui nomment également classèmes ces traits communs. Je le leur emprunte ici pour désigner, de manière générale, des traits relatifs à un signifié global. 
- dans le genre "éthique », une isotopie pouvant être notée [+SPÉCULATIVITÉ] (ou [-OBJECTIVITÉ]) s'accorde avec le fait qu'une proposition éthique, comme toute "vérité générale », ne possède pas le caractère arbitraire d'une loi : au contraire, spéculative, elle est motivée (par une représentation, un raisonnement, des expérimentations, etc.) et peut, eu égard à son caractère motivé, être reconnue, ou discutée, ou contestée, etc.; cette isotopie [+SPÉCULATIVITÉ] sous-tend la composition de la valeur flexionnelle [+vérité générale] du verbe naissent (objet d'une pensée spéculative) avec sa valeur lexicale [+destinée] (la destinée d'un individu, ou celle de l'espèce humaine, est un objet conceptuel spéculatif).

Les isotopies différentielles [+OBJECTIVTTÉ] et [+SPÉCULATIVITÉ] mises en évidence quant au verbe naissent lient semblablement la valeur flexionnelle et la valeur lexicale des deux autres verbes conjugués de l'article 1.

Quant au groupe verbal sont donés ${ }^{21}$,

- dans le genre « légal», l'isotopie [+OBJECTIVITÉ] sous-tend la composition de la valeur flexionnelle [+performatif légal] de ce groupe verbal avec une valeur lexicale [-agent implicite] - cette dernière, "adjectivale », correspondant à un emploi de doués constatant un fait sans se préoccuper de sa genèse (comme dans la phrase cet espace est doué de profondeur ${ }^{22}$ ) ; l'objectivité de la performativité légale de la DUDH s'accorde ici avec le caractère constatif (objectif) des qualités légiférées; on peut en outre considérer que, ce texte se bornant à signifier une vérité générale ("les êtres humains sont doués de raison et de conscience, c'est ainsi »), la valeur performative de sont doués revient à l'institutionnalisation légale de cette vérité générale - et le test du remplacement par un futur, attestant la valeur flexionnelle, prendra ce fait en considération : «ils seront considérés/reconnus comme doués... »);

- dans le genre "éthique », l'isotopie [+SPÉCULATIVTTÉ] sous-tend la composition de la valeur flexionnelle [+vérité générale] de ce groupe verbal avec une valeur lexicale [+agent implicite], cette dernière étant une valeur «verbale» de donés, héritant par connotation $^{23}$, contrairement à la valeur «adjectivale» du genre «éthique», de la structure argumentale du verbe doner à la voie passive - qui implique l'existence d'un complément d'agent, demeurant implicite en l'occurrence : «les êtres humains ont été doués de raison et de conscience (par un «agent»), c'est pourquoi ils sont doués de raison et de conscience $»$.

Quant au verbe doivent,

- dans le genre « légal », l'isotopie [+OBJECTIVTTÉ] sous-tend la composition de la valeur flexionnelle [+performatif légal] de ce verbe avec une valeur lexicale [+impératif légal] (ou [- impératif moral]) ; dans le genre «légal » en effet, la valeur déontique juridique portée par le temps verbal «présent »spécifie une valeur déontique non spécifiquement juridique portée par le radical de ce même verbe (c'est-à-dire son sens

\footnotetext{
21 On ici considère sont doués à parité avec une forme verbale dans la mesure où le verbe sont est, sémantiquement, un simple «support d'attribut». (Par ailleurs, on simplifie l'analyse en la limitant au cas où les propositions contenant les verbes sont doués et doivent sont interprétées comme des propositions sémantiquement indépendantes.)

22 ou « Le benzole est doué de propriétés antidétonantes », TLF : 466.

23 On pourrait encore parler, à ce propos, de valeur afférente ( $c f$. note infra).
} 
lexical d'auxiliaire modal) - le test de cette transformation étant que le présent de doivent peut, ici, être remplacé salva congruitate par un futur ;

- dans le genre « éthique », l'isotopie [+SPÉCULATIVTTÉ] sous-tend la composition de la valeur flexionnelle [+vérité générale] de doivent avec une valeur lexicale [+impératif moral] (ou [-impératif légal]) : dans le genre «éthique» en effet, cette valeur lexicale est celle d'un déontique ordinaire, non spécifiquement juridique - le test de cette valeur étant que le présent ne peut pas, ici, être remplacé par un futur.

\subsubsection{Le genre "éthique " s'oppose au genre "légal" par une ouverture de son sens}

L'ensemble des analyses qui précèdent concourt à montrer que le signifié global [+ETHIQUE] détermine un sens plus «ouvert» que le signifié global [+LOI]. Cette « ouverture » peut être remarquée :

- quant à la valeur flexionnelle (présent) des trois verbes conjugués de l'article $1:$ la valeur [+vérité générale] du genre "éthique ", non performative et prêtant à discussion, est plus ouverte que la valeur du genre « légal », performative et ne prêtant pas à discussion en tant que telle ;

- quant à la valeur lexicale [+destinée] du verbe naissent : celle-ci réfère dans le genre «éthique » à une qualification plus ouverte - parce que moins certaine - que son opposée qui dans le genre « légal » ne désigne qu'une qualité originaire objectivée ;

- quant à la valeur lexicale [+agent implicite] du groupe sont doués : référant dans le genre « éthique » à l'existence possible d'une cause - non explicitement déterminée des qualités prédiquées, cette valeur est plus ouverte que la valeur opposée dans le genre « légal » qui exclut cette référence ;

- quant à la valeur lexicale [+impératif moral] du verbe doivent: cette valeur, dans le genre «éthique », est plus ouverte - quant à la définition de l'impératif moral, auquel elle renvoie ici encore de manière non explicitement déterminée - que son opposée référant à un impératif légal parfaitement déterminé en tant que tel.

Si l'on analysait les valeurs des noms et des adjectifs de l'article 1, il apparaitrait que ces deux isotopies différentielles sont également agissantes pour la quasi totalité d'entre eux, et que leur sens est, semblablement, plus « ouvert » lorsqu'il est déterminé par le signifié global [+ETHIQUE] que lorsqu'il est déterminé par le signifié global [+LOI].

Ce que ces analyses mettent clairement en lumière, c'est que la DUDH interprétée comme une loi n'est pas, sémantiquement, le même texte que la DUDH interprétée comme un idéal éthique. Ce faisant, ces analyses suggèrent qu'une réflexion sur l'universalité ou la non-universalité de la Déclaration de 1948 ne saurait faire l'économie d'un examen préalable du problème d'interprétation soulevé - par un texte qui se présente lui-même, assez paradoxalement, comme un idéal éthique énoncé sous forme de loi. De fait, toute réflexion sur l'universalité ou la non-universalité qui ne serait pas attentive à la sémantique de la DUDH risquerait de ne pas porter sur un objet réel.

\subsubsection{Deux hypothèses relatives à la plasticité sémantique du genre "éthique "}

La linguistique de l'interprétation ne permet pas seulement d'attirer l'attention sur la pluralité possible des significations de la Déclaration de 1948. Cette linguistique 
peut également, par des analyses plus fines, contribuer à éclairer la question évoquée en introduction «La DUDH est-elle légitimement universelle, ou est-elle entachée d'ethnocentrisme ? » - en disqualifiant cette question pour la remplacer par une autre : "Quelles sont les conditions d'interprétation selon lesquelles la DUDH peut être comprise (ou non) comme universelle?».

Le fil conducteur propre à guider cette investigation sera fourni par l'analyse des isotopies différentielles (supra, 2.1.4.) établissant que le genre « éthique » détermine un sens plus ouvert que le genre «loi ». En effet, ce phénomène, qui peut être également qualifié de plasticité sémantique du genre «étbique», est propre à inspirer deux hypothèses :

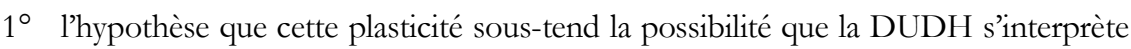
différemment en fonction d'arrière-plans culturels distincts ;

$2^{\circ}$ l'hypothèse que cette plasticité sous-tend également, conjointement, la possibilité d'une interprétation «non universelle » de la DUDH et celle de son interprétation « universelle ».

Ces hypothèses ne sont pas des spéculations métaphysiques : portant sur les conditions matérielles de l'interprétation du texte, elles doivent pouvoir être confirmées par l'analyse - c'est-à-dire par l'établissement de lois de corrélation entre signifiés globaux et signifiés locaux. Dans cette perspective, ces deux hypothèses pourront être examinées sur la base de deux subdivisions du signifié global [+ETHIQUE] (ces subdivisions s'entendant toujours, pour satisfaire au principe de différentialité, comme des bipartitions oppositives) :

$1^{\circ}$ sa subdivision par des traits différentiels successifs répondant de divers arrièreplans culturels ;

$2^{\circ}$ sa subdivision par des traits différentiels propres à rendre compte du caractère « universel » ou « non universel» de l'interprétation de la DUDH.

\subsection{Plasticité du genre "éthique " et diversité des arrière-plans culturels}

\subsubsection{Valeurs locales et valeurs globales}

Alors que ce sont des valeurs locales inhérentes qui ont été captées dans l'opposition des genres «loi » et «éthique » - c'est-à-dire : des valeurs inscrites (" dénotées ») dans le système différentiel de la langue -, il n'en ira pas de même pour les valeurs locales liées aux arrière-plans culturels de la DUDH. L'analyse identifiera cette fois, quant aux signifiés locaux, des valeurs afférentes - c'est-à-dire : des valeurs " connotées», ressortissant à d'autres normes que celles du système de la langue ${ }^{24}$. Lesdites valeurs afférentes, également différentielles, ne se composent pas moins avec les valeurs inhérentes des unités linguistiques auxquelles elles sont liées. Pour faire apparaitre cette composition, les analyses qui suivent s'appliqueront aux mêmes objets

\footnotetext{
24 Notre distinction sémantique inbérence/afférence s'inspire de celle de F. Rastier (cf. par exemple: Rastier, Cavazza et Abeillé, 1994 : 53).
} 
que les précédentes: les morphèmes flexionnels et les morphèmes lexicaux des trois verbes conjugués de l'article 1 .

Regardant le genre (la valeur globale) lié à un contexte culturel, on choisira, pour donner un exemple simple de ces analyses «culturelles", deux subdivisions successives du genre noté [+ETHIQUE] :

- la première subdivision divisera ce genre en deux genres, notés [+RELIGION] et [-RELIGION] ; par "éthique religieuse » ([+ETHIQUE, +RELIGION]), on entendra une pensée morale se posant comme dépendante d'un fait culturel dit religion, répondant d'un ensemble de croyances, de dogmes, de pratiques et de textes ; par «éthique non religieuse » ([+ETHIQUE, -RELIGION]), on entendra une pensée morale se posant comme indépendante du fait culturel dit religion;

- la seconde subdivision, subséquente à la précédente, divisera le genre « éthique religieuse » en deux genres notés [+CRÉATIONNISTE] et [-CRÉATIONNISTE]; par "éthique religieuse créationniste » ([+ETHIQUE, +RELIGION, +CRÉATIONNISTE]), on entendra que cette éthique est attachée à une religion posant un acte créateur à l'origine du cosmos, de la terre, de l'homme, etc. (par exemple : les monothéismes judaïque, chrétien ou islamique); par «éthique religieuse non créationniste» ([+ETHIQUe, +RELIGION, -CRÉATIONNISTE]), on entendra que cette éthique est attachée à une religion ne posant pas d'acte créateur à l'origine du cosmos, de la terre, de l'homme, etc. (par exemple : le bouddhisme).

\subsubsection{Exemples de corrélations déterminées par les gentes "éthique religieuse» et "éthique non religieuse "}

Les lois de corrélation différentielles des signifiés globaux [+ETHIQUE, +RELIGION] et [+ETHiQUe, -RELIGION] avec les signifiés locaux des verbes conjugués de l'article 1 décriront la composition des valeurs locales inhérentes du genre "éthique » (valeurs analysées supra, section 2) avec des valeurs afférentes propres aux genres «éthique religieuse » ou «éthique non religieuse ».

(1) Verbes naissent, sont (doués) et doivent: la valeur flexionnelle inhérente [+vérité générale] se compose

- dans le genre «éthique religieuse », avec la valeur flexionnelle afférente [+référée à une religion] (les trois propositions de l'article 1 énoncent « une vérité référée à une religion $\gg)$;

- dans le genre " éthique non religieuse », avec la valeur flexionnelle afférente [référée à une religion] (les trois propositions de l’article 1 énoncent «une vérité non référée à une religion »).

(2) Verbe naissent: la valeur lexicale inhérente [+destinée] se compose

- dans le genre "éthique religieuse », avec la valeur lexicale afférente [+référée à une religion] ${ }^{25}$ (la première proposition de l'article 1 concerne « une destinée ellemême référée à une religion »);

- dans le genre « "éthique non religieuse», avec la valeur lexicale afférente [référée à une religion] (la première proposition de l’article 1 concerne « une destinée non référée à une religion »).

${ }^{25}$ La Déclaration d'indépendance des Etats-Unis (1776) - ancêtre de la Déclaration des droits de l'homme et du citoyen (1789) et de la DUDH - énonçait : «Tous les hommes sont créés égaux ». 
(3) Groupe verbal sont donés : la valeur lexicale inhérente [+agent implicite] se compose

- dans le genre «éthique religieuse » avec la valeur lexicale afférente [+(agent) défini par une religion] (la deuxième proposition de l'article 1 concerne « des qualités octroyées par un agent défini par une religion $»)^{26}$;

- dans le genre "éthique non religieuse », avec la valeur lexicale afférente [-(agent) défini par une religion] (la deuxième proposition de l'article 1 concerne « des qualités non octroyées par un agent défini par une religion »).

(4) Verbe doivent: la valeur lexicale inhérente [+impératif moral] se compose

- dans le genre " éthique religieuse », avec la valeur lexicale afférente [+énoncé par une religion] (la troisième proposition de l'article 1 « concerne un impératif moral énoncé par une religion »);

- dans le genre "éthique non religieuse », avec la valeur lexicale afférente [énoncé par une religion] (la troisième proposition de l'article 1 « concerne un impératif moral non énoncé par une religion»).

\subsubsection{Exemples de corrélations déterminées par les genres "éthique religieuse créationniste " et " éthique religieuse non créationniste "}

Les lois de corrélation différentielles des signifiés globaux [+ETHIQUE, +RELIGION, +CRÉATIONNISTE] et [+ETHIQUE, +RELIGION, -CRÉATIONNISTE] avec les signifiés locaux des mêmes verbes décriront la composition des valeurs locales inhérentes et afférentes du genre «éthique religieuse» (valeurs analysées supra, 2.2.2.) avec des valeurs afférentes propres aux subdivisions « créationniste » ou « non créationniste ».

(1) Verbes naissent, sont (doués) et doivent: les valeurs flexionnelles inhérente [+vérité générale] et afférente [ + référée à une religion] se composent

- dans le genre «créationniste », avec la valeur lexicale afférente [+créationniste] (la première proposition de l'article 1 concerne «une vérité référée à une religion créationniste »);

- dans le genre «non créationniste », avec la valeur lexicale afférente [-créationniste] (la première proposition de l'article 1 concerne « une vérité référée à une religion non créationniste »).

(2) Verbe naissent: les valeurs lexicales inhérente [+destinée] et afférente [+référée à une religion] se composent

- dans le genre «créationniste », avec la valeur lexicale afférente [+dessein d'un Créateur] (la première proposition de l'article 1 concerne « une destinée référée à une religion qui la conçoit comme le dessein d'un Créateur »).

- dans le genre "non créationniste », avec la valeur lexicale afférente [-dessein d'un Créateur] (la première proposition de l'article 1 concerne « une destinée référée à une religion qui ne la conçoit pas comme le dessein d'un Créateur »).

(3) Groupe verbal sont donés: les valeurs lexicales inhérente [+agent implicite] et afférente [+défini par une religion] se composent

\footnotetext{
${ }^{26}$ Dans la Déclaration d'indépendance des États-Unis, l'emploi de sont doués appartient explicitement à ce genre : «tous les hommes (...) sont doués par le Créateur de certains droits inaliénables».
} 
- dans le genre "créationniste », avec la valeur lexicale afférente [+Créateur] (la deuxième proposition de l'article 1 concerne «des qualités octroyées par un agent défini par une religion comme étant un Créateur»);

- dans le genre "non créationniste », avec la valeur lexicale afférente [-Créateur] (la deuxième proposition de l'article 1 concerne «des qualités octroyées par un agent défini par une religion non comme n'étant pas un Créateur $\left.{ }^{27} »\right)$.

(4) Verbe doivent: les valeurs lexicales inhérente [+impératif moral] et afférente [+énoncé par une religion] se composent

- dans le genre « créationniste », avec la valeur lexicale afférente [+dessein d'un Créateur] (la troisième proposition de l'article 1 concerne « un impératif moral énoncé par une religion et reflétant le dessein d'un Créateur »);

- dans le genre «non créationniste », avec la valeur lexicale afférente [-dessein d'un Créateur] (la troisième proposition de l'article 1 concerne « un impératif moral énoncé par une religion, et ne reflétant pas le dessein d'un Créateur »).

\subsubsection{Spécificité de la plasticité " culturelle "}

Notre première hypothèse sur la plasticité du genre «éthique », supposant une multiplication des interprétations de la DUDH selon ses arrière-plans culturels, se trouve confirmée par les corrélations qu'on vient de décrire.

Sur la base de ces exemples, on peut également définir, relativement à ce cas particulier des subdivisions «culturelles » du genre «éthique », le principe fonctionnel de ce que nous nommons plasticité sémantique. Cette plasticité obéit aux critères spécifiques suivants :

$1^{\circ}$ le signifié global «éthique » de la DUDH se laisse aisément subdiviser en de multiples sous-genres ;

$2^{\circ}$ ces sous-genres sont en nombre indéterminé (et ils ne revêtent aucune valeur absolue: ainsi, au lieu des sous-genres proposés ci-dessus, il aurait été parfaitement possible de subdiviser le signifié global [+RELIGION] en [+THEISME] / [-THEISME], puis le signifié global [+THEISME] en [+MONOTHEISME] / [MONOTHEISME], etc.) ;

$3^{\circ}$ les valeurs des signifiés locaux, dans tous les cas analysés ici, sont des valeurs afférentes.

En outre, une conclusion s'impose: nos analyses d'interprétations multiples correspondant aux sous-genres «culturels» ne sauraient gager une interprétation « universelle » de la DUDH. Mettant en lumière des valeurs oppositives qui sont par définition sémantiquement exclusives les unes des autres, ces analyses attestent au contraire, de facto, des interprétations propres à une culture, et en cela «non universelles $»^{28}$.

\footnotetext{
27 Dans le bouddhisme, cet agent sera une «loi de causalité », étendue ou non, suivant les écoles, à des mondes supranormaux.

${ }^{28}$ Certes, on peut argumenter que, se prêtant à ces multiples interprétations « culturelles », la DUDH est susceptible de jouer un rôle médiateur entre ces interprétations lorsqu'elle suscite des afférences sémantiques similaires ; mais ce rôle, s'il est lié à une illusoire "présomption d'universalité », peut tout aussi bien occasionner des malentendus - pouvant porter, par exemple, sur des conceptions
} 


\subsection{Plasticité du genre "éthique » et question de l'universalité}

\subsubsection{Signifié global : la dichotomie statique/dynamique}

Notre seconde hypothèse - supposant une plasticité sémantique propre à générer conjointement, dans le genre «éthique» de la DUDH, une interprétation « non universelle » et une interprétation « universelle » - ne saurait être argumentée sur la base du principe de plasticité «culturelle» puisque celui-ci ne sous-tend a priori, comme on l'a vu, que des interprétations « non universelles ». Cette seconde hypothèse doit donc s'étayer d'un principe de plasticité distinct, dont les critères programmatiques suivants peuvent être posés :

$1^{\circ}$ l'opposition «non universel»/ « universel» sera captée par une unique subdivision de signifié global (ou genre);

$2^{\circ}$ cette subdivision devra se combiner avec celles des multiples genres « culturels »;

$3^{\circ}$ la détermination des signes locaux par le signifié global «universel» devra consister en une neutralisation des valeurs oppositives attachées aux genres « non universels » régissant ces mêmes signes locaux.

Il se trouve que la réflexion développée par Henri Bergson dans Les deux sources de la morale et de la religion (Bergson, 1932), reprise par Karl Popper dans La société ouverte et ses ennemis (Popper, 1945), est en mesure de donner un contenu intuitif à ces critères programmatiques. La philosophie sociale bergsonienne, en effet, caractérise toute pensée éthique comme découlant de deux « sources » antagoniques et complémentaires :

- une «source» dite statique - ou encore institutionnelle -, d'où procède une organisation de pensée close (une pensée incarnée dans des institutions et exclusivement vouée à la conservation du statu quo d'un groupe social) ;

- une «source» dite dynamique - ou encore mystique (ce concept ne s'entendant pas comme limité au fait religieux) -, produisant une organisation de pensée ouverte (une pensée incarnée par des «sages » et liée, au contraire de la précédente, à un « élan créateur» compatible avec des valeurs plus universelles).

Alors que nous avons utilisé jusqu'ici le terme d'universel sans en problématiser la signification - nous référant simplement au fait que ce terme figure dans le titre de la Déclaration et qu'il est au centre de nombreux débats -, la dichotomie bergsonienne permet de préciser la dualité non universel / universel sur laquelle porte notre seconde hypothèse. En effet, noter cette dualité par les signifiés globaux [+STATIQUE] (équivalent de [-DYNAMIQUE]) et [+DYNAMIQUE] (équivalent de [-STATIQUE]) présente les avantages suivants :

- le trait [+STATIQUE] rendra compte de l'interprétation «culturelle » de la DUDH telle que nous l'avons analysée (supra, 2.2.), déterminant des valeurs «non universelles » opposées, exclusives les unes des autres : il s'agira, en l'occurrence, d'une interprétation conceptuellement figée, objectivée par une tradition culturelle, et vouée à la conservation d'un statu quo social ;

incompatibles de l'éthique (ou de la loi sous-tendue par cette éthique), ou encore sur des représentations divergentes des concepts attachés à des mots comme dignité, liberté, arbitraire, etc. 
- le trait [+DYNAMIQUE] permettra de capter un autre aspect de l'interprétation de la DUDH (dont notre analyse n'a jusqu'ici pas rendu compte), susceptible de se greffer sur n'importe quelle interprétation «statique »: il s'agira d'une interprétation conceptuellement « ouverte », correspondant à une " expérience de pensée » subjective (ce que Bergson nomme mystique), et visant une transformation éthique, un devenir (ce qu'on peut appeler transcendance). De fait, cette interprétation «dynamique » ne produira pas un sens universel en soi: ce sera plutôt un sens constitué de telle sorte que sa visée puisse être tenue pour l'objet possible d'une unanimité.

En bref, alors que l'interprétation "statique », dans ses subdivisions « culturelles », rendra compte de divergences de pensée, l'interprétation «dynamique» rendra compte, sur la base d'une plasticité sémantique, d'une convergence possible de pensée. Ces deux interprétations antagonistes - une doxa et une mystique - n'en seront pas moins complémentaires l'une de l'autre, en cela que, si l'immanence statique est bien distincte de la transcendance dynamique, la seconde n'en repose pas moins, chronologiquement et logiquement, sur la première.

Tout ceci, de nouveau, n'est pas pure spéculation métaphysique mais élaboration d'une hypothèse propre à être avérée par l'analyse interprétative.

\subsubsection{L'interprétation " dynamique » comme neutralisation des oppositions de l'interprétation "statique "}

La préséance chronologique et logique de l’interprétation «statique »sur l'interprétation "dynamique », qu'on vient de postuler, implique, en termes sémantiques, de poser le «sens ouvert» de l'interprétation «dynamique » comme une neutralisation des oppositions de l'interprétation «statique ». Pour illustrer par quelques exemples comment le signifié global [+DYNAMIQUE] peut neutraliser les oppositions de valeurs locales déterminées par le signifié global [+STATIQUE], on reprendra l'analyse menée plus haut sur les genres notés [+ETHIQUE, +RELIGION] et [+ETHIQUE, -RELIGION], en poursuivant la subdivision de chacun de ces signifiés globaux par la dualité [+STATIQUE] / [+DYNAMIQUE].

(1) Verbes naissent, sont (doués) et doivent-composition des valeurs flexionnelles inhérente [+vérité générale] et afférentes [+référée à une religion] ou [-référée à une religion] (ainsi que toutes leurs subdivisions possibles) :

- dans l'interprétation "statique », ces valeurs se composent avec une valeur afférente [+sens fermé] (les trois propositions de l'article 1 réfèrent à une vérité close, objectivée dans une pensée instituée, conservatrice d'un statu quo social);

- dans l'interprétation «dynamique », ces valeurs se composent avec une valeur afférente [+sens ouvert] (les trois propositions de l'article 1 réfèrent à une vérité ouverte, relevant d'une expérience de pensée de type mystique (au sens de Bergson) et d'une visée unanime possible ${ }^{29}$ - cette vérité étant, de ce fait, à la fois distincte et non exclusive de celle de l'interprétation « statique »).

Ces traits afférents [+sens fermé] et [+sens ouvert] de la flexion des trois verbes se composeront, par isotopie, avec les compositions des valeurs lexicales desdits verbes.

\footnotetext{
${ }^{29}$ L'« ouverture », ici, ne renvoie pas à une relativité de cette interprétation mais au fait qu'elle reflète le dynamisme d'une expérience et un devenir de pensée. Cette valeur flexionnelle s'attachant à une "vérité heuristique » conditionne, comme on le verra, les valeurs lexicales.
} 
(2) Verbe naissent - composition des valeurs lexicales inhérente [+destinée] et afférentes [+référée à une religion] ou [-référée à une religion] (ainsi que leurs subdivisions) :

- dans l'interprétation "statique», ces valeurs se composent avec la valeur afférente [+sens fermé] (la « destinée » humaine est une notion close, objectivée dans une pensée instituée, et conservatrice de statu quo);

- dans l'interprétation "dynamique», ces valeurs se composent avec la valeur afférente [+sens ouvert] (la « destinée » humaine est une notion ouverte, appartenant à une expérience de pensée subjective - dans laquelle elle peut prendre un sens transcendant, non attaché exclusivement à une pensée instituée, sans pour autant exclure aucune pensée instituée).

(3) Groupe verbal sont doués - composition des valeurs lexicales inhérente [+agent implicite] et afférentes [+défini par une religion] ou [-défini par une religion] (ainsi que leurs subdivisions):

- dans l'interprétation "statique», ces valeurs se composent avec la valeur afférente [+sens fermé] (connotant un «agent» identifié dans des institutions de pensée, religieuses ou non religieuses - par exemple : Jéhovah, Dieu ou Allah, dans les dogmes judaïque, chrétien ou islamique ; dans un dogme athée, l'évolution per se);

- dans l'interprétation "dynamique», ces valeurs se composent avec la valeur afférente [+sens ouvert] (l'agent implicite de la deuxième proposition de l'article 1 est un principe non objectivable, qui excède le pensable; dans cette interprétation la valeur [+défini par une religion], par exemple, cesse d'être exclusive de son opposée, et inversement).

(4) Verbe doivent-composition des valeurs lexicales inhérente [+impératif moral] et afférentes [+énoncé par une religion] ou [-énoncé par une religion] (ainsi que leurs subdivisions) :

- dans l'interprétation «statique », ces valeurs se composent avec la valeur afférente [+sens fermé] ('impératif moral est figé, objectivé dans une pensée instituée, conservateur de statu quo);

- dans l'interprétation «dynamique», ces valeurs se composent avec la valeur afférente [+sens ouvert] (l'impératif moral est ouvert, nourri par une expérience de pensée subjective dans laquelle il peut devenir l'objet d'une visée unanime).

Comme on en a fait l'hypothèse, l'interprétation «dynamique » atteste bien, sur la base d'une plasticité sémantique, une convergence possible de pensée - là où l'interprétation «statique » et ses subdivisions «culturelles » attestaient des divergences. La plasticité "dynamique ", ainsi qu'on l'a vu, neutralise les divergences de l'interprétation «statique »; elle ne les abolit pas pour autant puisque, d'une part, la convergence requiert ici le point de départ des divergences et que, d'autre part, sa neutralisation n'est pas une suppression mais une sorte de "mise entre parenthèses » heuristique au service d'une interprétation transcendante. Le paradoxe de l'universalité, conçue comme interprétation «dynamique», est que l'éthique de la DUDH est d'autant plus pensable comme «universelle » que, sur le fondement d'un 
sens ouvert, elle est propre à accueillir les différences culturelles sans rien niveler de ces différences ${ }^{30}$.

Finalement, le concept de transcendance, dans l'usage que nous en avons fait, réfère à la qualité d'un type particulier de plasticité sémantique, rien de plus. Il en va de même du qualificatif bergsonien de mystique attribué ici à l'éthique. Et, dans cette perspective, le «sens ouvert» d'une interprétation éthique «dynamique » est comparable au «sens ouvert» en poésie. Cette parenté est attestée par un fait remarquable : de même qu'un texte poétique, traduit dans une langue quelconque, perd des particularités de sa langue originaire mais conserve intact son caractère voire sa force de poéticité, de même un texte comme la DUDH dans son interprétation "éthique » peut être traduit dans toutes les langues et compris dans toutes les cultures en conservant intact un caractère et une force d'éthicité.

\subsection{Enjeux d'une sémantique des droits de l'homme}

Les analyses présentées ici ne font qu'esquisser une sémantique de la DUDH. Elles n'en laissent pas moins entrevoir que cette sémantique est probablement en mesure de clarifier et de soutenir deux enjeux socio-politiques :

$1^{\circ}$ combattre et disqualifier les instrumentalisations de la DUDH ;

$2^{\circ}$ éclairer la compréhension du rôle pouvant être joué par l'unanimité dans le domaine de la gouvernance mondiale.

\subsubsection{Combattre les instrumentalisations de la DUDH}

L'instrumentalisation politicienne de la DUDH consiste notamment à clamer son universalité, ou au contraire à contester celle-ci, dans le but de servir des intérêts hégémonistes ou totalitaires. Ces manœuvres ont en commun une réification du sens: qu'elles s'en prévalent ou qu'elles l'attaquent, elles conçoivent la DUDH comme univoque - comme pourvue d'un sens existant en lui-même et par lui-même.

L'analyse d'une linguistique de l'interprétation fait voler en éclats cette réification du sens. Dissipant l'illusion d'un texte univoque et montrant que le sens de la Déclaration n'est pas figé en lui-même mais produit par des interprétations multiples, elle invalide aussi bien la thèse d'une pseudo-universalité insoucieuse des diversités culturelles que la thèse d'une pseudo-non-universalité réfractaire à toute visée convergente. Ainsi, la sémantique de la DUDH disqualifie-t-elle par avance l'instrumentalisation hégémoniste ou totalitariste. L'approche sémantique peut également servir à faire la part entre les diverses lectures et les instrumentalisations possibles de textes comme la Charte africaine des droits de l'homme et des peuples (1981), la Déclaration islamique universelle des droits de l'homme (1981) ou la Déclaration de Bangkok (1993) - des textes qui se prêtent à être interprétés tout autant dans un genre «statique » que dans un genre "dynamique». Et cette approche vaut semblablement, a priori, pour tout texte à venir.

30 «Il faudra la diversité dont nous avons tant besoin. Une des valeurs cibles de la spiritualité commune, c'est la préservation la diversité. » (S. Hessel, in Bouquet et Hessel, 2012 : 12) 
En résumé, l'approche sémantique permet de dénoncer les instrumentalisations de la DUDH et des textes de sa parenté, en analysant ces instrumentalisations comme reposant sur une interprétation « statique » ad hoc.

\subsection{2. Éclairer la perspective de l'unanimité dans une gouvernance mondiale}

L'analyse de l'interprétation «dynamique » permet, quant à elle, d'accréditer une lecture de la DUDH pouvant être effectivement dite universelle. Il est toutefois plus clair de qualifier cette lecture de possiblement unanime car, comme on l'a vu (supra, 2.3.2.), elle se soutient d'un processus interprétatif gageant une unanimité possible et non un sens ordinaire ; en d'autres termes, ce n'est pas la DUDH en tant que sens établi qui a la qualité d'« universalité » ou de transcendance, mais une interprétation de la DUDH en tant qu'aspiration ${ }^{31}$. L'enjeu d'identifier une telle sémantique est d'importance, en cela qu'il y va de la mise en évidence d'une lecture unanime possible d'un texte fondateur de l'ONU, tout autant que de la conception d'une transcendance compatible avec la diversité culturelle.

Mais l'analyse de l'interprétation «dynamique » de la DUDH peut également jouer un rôle critique au regard des institutions de l'ONU $^{32}$. En effet, mettant en lumière les conditions interprétatives de l'unanimité possible, et posant ces dernières comme fondatrices du fait même de l'unanimité possible, cette analyse montre que l'unanimité interprétative n'est accessible qu'au prix de l'organisation de pensée que Bergson nomme, techniquement, mystique - correspondant, dans une terminologie sémantique, à une ouverture du sens. De cette organisation de pensée, les institutions de l'ONU peuvent-elles être les garantes, tant pour interpréter la DUDH et les textes de sa parenté, que pour éventuellement les compléter et les adapter au cours du temps? Dans une perspective bergsonienne, la réponse semble être non, car l'interprétation ressortissant au genre "dynamique» ne peut, selon la définition même de ce genre, être gagée sur des institutions: cette interprétation et ses valeurs sont gagées, par définition, sur la dimension «mystique» de traditions humaines de réflexion, de spiritualité, de sagesse. Aussi semblerait-il qu'une articulation reste à trouver entre le gage institutionnel et un gage «mystique» de la DUDH. Une articulation dont l'analyse sémantique pourrait stimuler, à sa manière, la compréhension.

\footnotetext{
31 Si l'unanimité peut être ainsi posée à la base de l'analyse sémantique «dynamique » de la DUDH, elle est aussi sa fondation historique, sous la forme minimale du vote sans opposition des États membres de l'ONU qui l'a instituée le 10 décembre 1948.

32 «Je pense que nous avons besoin d'une réflexion approfondie sur la légitimité des institutions qui s'inspirent peut-être vaguement de la Déclaration universelle mais qui se sont beaucoup différenciées. » (S. Hessel, in Bouquet et Hessel, 2012 : 12)
} 
Simon Bouquet

\section{BIBLIOGRAPHIE}

Bergson, H., 1932, Les deux sources de la morale et de la religion, Paris, Presses universitaires de France.

BOUQUET, S., 2011, «Da una epistemologia neosaussuriana della linguistica alla questione dell'universalità dei diritti dell'uomo », Saussure, Quaderni di Rivista Italiana di Filosofia del Linguaggio, Catania, Bonanno Editore.

Bouquet, S., 2012, «Principes d'une linguistique de l'interprétation. Une épistémologie néosaussurienne », L'apport des manuscrits de Ferdinand de Saussure, Langages, n 185, p. 21-33.

Bouquet, S. et Hessel, S., 2012, « Pour une linguistique des droits de l'homme » (entretien de Stéphane Hessel avec Simon Bouquet, Collegium International, 22 février 2010), Texto! Revue électronique de l'Institut Ferdinand de Saussure, vol. XVII, p. 1-12.

Cossuta, F. et Maingueneau, D., 1995, «L'analyse des discours constituants », Les analyses du discours en France, Langages, n ${ }^{\circ} 117$, p. 112-125.

LEEMAN, D. (éd.), 2011, L'interculturel en entreprise : quelles formations? Aspects civilisationnels, économiques, bistoriques, juridiques, linguistiques, Limoges, Lambert-Lucas.

POPPER, K., 1945, The Open Society and Its Enemies, London, Routledge.

Rastier, F., Cavazza, M., ABeillé, A., 1994, Sémantique pour l'analyse, Paris, Masson.

SAussure (de), F., 1968, Cours de linguistique générale, édition critique par R. Engler, Wiesbaden, Otto Harrassowitz.

SAussure (de), F., 2002, Écrits de linguistique générale, édités par S. Bouquet et R. Engler, Paris, «Bibliothèque de Philosophie », Gallimard. 